\title{
LGR5 expression is associated with prognosis in poorly differentiated gastric adenocarcinoma
}

Takehito Ehara', Takeshi Uehara2 ${ }^{2 *}$, Tomoyuki Nakajima², Yasuhiro Kinugawa ${ }^{2}$, Shota Kobayashi' ${ }^{2}$ Mai Iwaya ${ }^{2}$, Hiroyoshi Ota ${ }^{2,3}$ and Yuji Soejima'

\begin{abstract}
Background: Leucine-rich repeat-containing G-protein-coupled receptor 5 (LGR5) is an important cancer stem cell marker in gastric cancer. However, no detailed studies are available on LGR5 expression in poorly differentiated gastric adenocarcinoma (PD-AC). Therefore, we investigated the relationship between LGR5 expression and clinicopathological data in PD-AC.
\end{abstract}

Methods: LGR5 mRNA expression levels were quantified in 41 PD-AC specimens using a highly sensitive RNAscope in situ hybridization technique. Epstein-Barr virus (EBV) infection was also detected by EBV in situ hybridization.

Results: LGR5 expression levels were measured in 38 of 41 PD-AC cases, and 17 cases were identified as LGR5 high. The frequency of EBV positivity tended to be higher in the LGR5-low group than in the LGR5-high group ( $P=$ 0.0764). Furthermore, the frequency of vascular invasion tended to be higher in the LGR5-high group than in the LGR5-low group $(P=0.0764)$. The overall survival of PD-AC patients in the $L G R 5$-high group was significantly lower than in the LGR5-low group (log-rank test, $P=0.0108$ ). The Cox proportional hazard regression model revealed that the LGR5-low group ( $\mathrm{HR}=0.29 ; 95 \% \mathrm{Cl}: 0.11-0.74 ; P=0.01$ ) showed independently better OS for PD-AC.

Conclusions: Quantifying the levels of LGR5 expression may facilitate defining prognosis in Japanese patients with PD-AC. Further study of LGR5 in this context is warranted.

Keywords: Leucine-rich repeat-containing G-protein-coupled receptor 5, Poorly differentiated gastric adenocarcinoma, RNA in situ hybridization

\section{Background}

Although the incidence of gastric cancer is decreasing, many individuals develop the disease. Gastric cancer is the fifth most frequent cancer and the third leading cause of cancer death [1]. Various treatment methods for gastric cancer, including surgery, radiation, and chemotherapy, have been performed. Among them, many recent studies have been conducted on cancer

\footnotetext{
* Correspondence: tuehara@shinshu-u.ac.jp

${ }^{2}$ Department of Laboratory Medicine, Shinshu University School of Medicine, 3-1-1 Asahi, Matsumoto 390-8621, Japan

Full list of author information is available at the end of the article
}

stem cells (CSCs), which are present in tumor tissues, and anti-CSC therapies have shown promising results [2]. CSCs are resistant to radiation and chemotherapy, and residual CSCs contribute to tumor regrowth. Therefore, effective anti-CSC therapy should be administered in combination with existing methods, such as chemotherapy and radiotherapy [3]. Some reports have investigated gastric CSCs, which typically express CD44, CD133, and Musashi-1 [4]. Additionally, leucine-rich repeat-containing G-proteincoupled receptor 5 (LGR5) was identified as a robust CSC marker in murine gastric cancer $[5,6]$.

(C) The Author(s). 2021 Open Access This article is licensed under a Creative Commons Attribution 4.0 International License, which permits use, sharing, adaptation, distribution and reproduction in any medium or format, as long as you give appropriate credit to the original author(s) and the source, provide a link to the Creative Commons licence, and indicate if changes were made. The images or other third party material in this article are included in the article's Creative Commons licence, unless indicated otherwise in a credit line to the material. If material is not included in the article's Creative Commons licence and your intended use is not permitted by statutory regulation or exceeds the permitted use, you will need to obtain permission directly from the copyright holder. To view a copy of this licence, visit http://creativecommons.org/licenses/by/4.0/ The Creative Commons Public Domain Dedication waiver (http://creativecommons.org/publicdomain/zero/1.0/) applies to the data made available in this article, unless otherwise stated in a credit line to the data. 
The standard treatment for stage I gastric cancer is curative resection only, and it is associated with an excellent prognosis [7]. On the other hand, the standard treatment for stage II/III gastric cancer is curative resection and adjuvant chemotherapy, but the frequency of recurrence is high [8]. Therefore, it is necessary to improve the prognosis of stage II/III gastric cancer patients. Furthermore, the components of poorly differentiated cancer tissues also have a significant impact on prognosis. In gastric cancer, poorly differentiated adenocarcinoma (PD-AC) has a poor prognosis [9], but the underlying mechanisms remain unclear. Additionally, the expression of CSC markers in poorly differentiated gastric cancer, especially LGR5, has not been reported. Therefore, we investigated the clinicopathological relationship between LGR5 marker expression and prognosis in stage II/III gastric cancer patients.

\section{Methods}

\section{Patients and materials}

We identified 91 PD-AC cases who underwent surgical resection between 2008 and 2018 at six institutes [Shinshu University Hospital (Matsumoto, Japan), Nagano Municipal Hospital (Nagano, Japan), Aizawa Hospital (Matsumoto, Japan), Showa Inan General Hospital (Komagane, Japan), Iida Municipal Hospital (Iida, Japan), and Nagano Matsushiro General Hospital (Nagano, Japan)] and evaluated their clinicopathological features. Of these patients, stage II and III cases were selected. A re-evaluation by two pathologists (T.U. and H.O.) before analysis excluded five cases that did not contain poorly differentiated components, and 41 cases remained as candidates for analysis. This study was approved by the Ethics Committee of Shinshu University, Japan (no. 4088).

\section{Histopathology, immunohistochemical staining, and evaluation}

Paraffin blocks fixed with $8 \%$ formaldehyde containing sufficient tumor for analysis were prepared for hematoxylin and eosin (HE) staining and tissue microarray (TMA) analysis by extracting a core with a diameter of $3 \mathrm{~mm}$ from each case. Additionally, the TMA was subjected to immunostaining using antibodies against the following mismatch repair proteins (MMRPs): MLH1 (ES05; mouse monoclonal; dilution 1:50), PMS2 (EP51; rabbit monoclonal; dilution 1:40), MSH2 (FE11; mouse monoclonal; dilution 1:50), or MSH6 (EP49; rabbit monoclonal; dilution 1:50; Agilent Technologies, Santa Clara, CA, USA), as described previously [10]. Representative images of the slides were captured with an Olympus DP74 camera (lens, $\times$ 40; Olympus, Tokyo, Japan) using the CellSens Standard software (Olympus, Tokyo, Japan). The images were acquired at a resolution of $96 \mathrm{dpi}$ and Adobe Photoshop was used to enhance the resolution of the images to $300 \mathrm{dpi}$. As reported in our previous paper, the staining results were scored as positive when a nuclear staining pattern was observed. If at least one of the four antibodies did not show expression, MMR protein deficiency was indicated. In the tumor, the tumor-infiltrating lymphocyte (TIL) score was assessed using a four-tier scale and recorded as follows: none: 0; mild: 1; moderate: 2; and marked: 3 [11]. The TIL score was classified as low grade (scores 0 and 1 ) and high grade (2 and 3).

\section{EBER in situ hybridization}

The EBER in situ hybridization assay was performed on TMA block sections. Epstein-Barr virus (EBV) was identified using EBER probes (ISH iVIEW Blue detection kit; Ventana Medical Systems Inc., Oro Valley, AZ, USA).

\section{LGR5 RNA in situ hybridization}

LGR5 mRNA detection was performed using the RNAscope $^{\bullet}$ kit (Advanced Cell Diagnostics, Hayward, CA, USA), as described previously [10]. Additionally, a fourstep evaluation method we reported previously was used [10]. Furthermore, LGR5 mRNA expression levels were categorized as low expression (grades $0,1+, 2+$, and $3+$ ) and high expression (4+). We analyzed the relationship between $L G R 5$ expression levels and the clinicopathological data and prognosis in patients with PD-AC, particularly regarding overall survival (OS).

\section{TCGA analysis}

mRNA-seq analysis was performed using the TCGA database. The TCGA data were downloaded from cBioPortal (http://www.cbiopor- tal.org/) in the form of mRNA median values. All clinical and pathological data for the TCGA cohort were downloaded from cBioPortal. The TCGA cohort was classified into high or low LGR5 mRNA expression groups. LGR5 high indicates $L G R 5$ mRNA expression $\geq$ the median, and LGR5 low indicates $L G R 5$ mRNA expression $<$ the median. Stage II/III patients were included in the first analysis, and then only Stage II/III patients with histological grade 3 were included in the second analysis.

\section{Statistical analysis}

The chi-squared test was applied to assess statistical significance. A $P$-value $<0.05$ was considered to be statistically significant. The OS rates of PD-AC patients were calculated using the Kaplan-Meier method, and differences were compared using the log-rank test. Univariate and multivariate analyses for prognostic factors were performed using the Cox proportional hazard regression model. A $P$-value $<0.05$ was considered to be statistically significant. Statistical analysis was performed using JMP version 13 (SAS Institute Japan, Tokyo, Japan). 


\section{Results}

LGR5 expression in PD-AC

In PD-AC patients, 38 of 41 cases showed LGR5 expression. Among them, 17 cases were identified as LGR5 high (Fig. 1a and b). Moreover, LGR5 expression was completely absent in three cases (Fig. 1d and e). LGR5 expression varied from diffuse to scattered.

\section{Relationship between LGR5 expression and clinicopathological characteristics}

EBER expression was negative in most cases (Fig. 1c). Although there were few EBV-positive PD-AC cases (Fig. 1f), all exhibited low LGR5 expression. LGR5 expression and clinicopathologic data are shown in Table 1. The number of EBV-positive PD-AC cases tended to be higher in the LGR5-low expression group than in the $L G R 5$-high expression group $(P=0.0764)$. The frequency of vascular invasion tended to be higher in the LGR5high expression group than in the LGR5-low expression group $(P=0.0764)$. No significant difference was found between the LGR5-high expression group and LGR5-low expression group regarding TILs, MSI, histological subtype, or TNM stage.

\section{Prognostic value of LGR5 in PD-AC}

The prognostic value of $L G R 5$ expression in PD-AC was analyzed by the Kaplan-Meier method and log-rank test (Fig. 2). The median OS for the study patients was 1146 (range; 635.5-1718) days. A significant difference was found in OS between PD-AC cases in the LGR5-high expression group [median OS: 756 (range; 154.5-1306.5) days] and LGR5-low expression group [median OS: 1338 (range; 922.75-2022.75) days] (log-rank test, $P=0.0108$ ).

We also compared the first quartile with grades $0-2$ and the fourth quartile with grade 4 . A significant difference was found in OS between PD-AC cases in the first and fourth quartiles (log-rank test, $P=0.0072$ ).

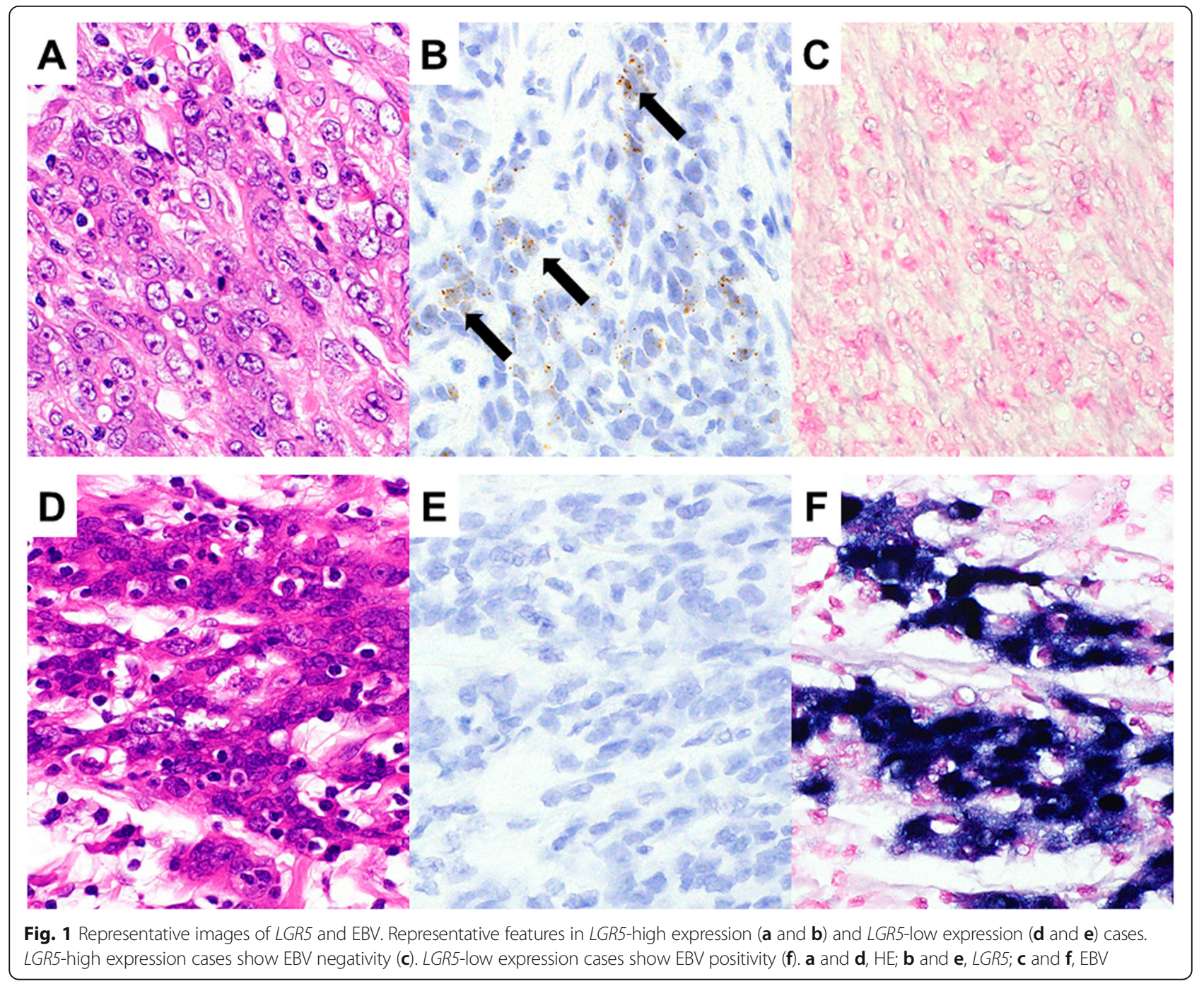


Table 1 LGR5 expression and clinicopathological characteristics in PD-AC

\begin{tabular}{|c|c|c|c|c|}
\hline \multirow[t]{2}{*}{ Factors } & \multicolumn{4}{|c|}{ LGR5 expression } \\
\hline & $\mathrm{n}$ & High $(n=17)$ & Low $(n=24)$ & $P$-value \\
\hline Age & & & & 0.0472 \\
\hline$>74$ years & 19 & 11 & 8 & \\
\hline$\leq 74$ years & 22 & 6 & 16 & \\
\hline Sex & & & & 0.0498 \\
\hline Male & 24 & 13 & 11 & \\
\hline Female & 17 & 4 & 13 & \\
\hline EBV & & & & 0.0764 \\
\hline Positive & 4 & 0 & 4 & \\
\hline Negative & 37 & 17 & 20 & \\
\hline Vascular invasion & & & & 0.0764 \\
\hline Present & 37 & 17 & 20 & \\
\hline Absent & 4 & 0 & 4 & \\
\hline TIL & & & & 0.283 \\
\hline High & 25 & 9 & 16 & \\
\hline Low & 16 & 8 & 8 & \\
\hline MSI & & & & 0.2176 \\
\hline Present & 19 & 10 & 9 & \\
\hline Absent & 21 & 7 & 14 & \\
\hline Differentiated-type & & & & 0.2921 \\
\hline Solid-type 1 & 8 & 2 & 6 & \\
\hline Non-solid-type 2 & 33 & 15 & 18 & \\
\hline TNM stage & & & & 0.9382 \\
\hline$\|$ & 19 & 8 & 11 & \\
\hline III & 22 & 9 & 13 & \\
\hline
\end{tabular}

We evaluated the relationship between clinicopathological factors and LGR5 expression regarding OS using a Cox proportional hazard regression model (Table 2), which revealed that the LGR5-low expression group (HR $=0.29$; 95\% CI: 0.11-0.74; $P=0.01$ ) had independently better $\mathrm{OS}$ for PD-AC.

\section{TCGA data analysis}

First, we examined the LGR5 mRNA expression levels in the TCGA cohort of stage II/III patients. There was no significant difference in OS between the LGR5 high and low groups (log-rank test, $P=0.7175$ ). Univariate and multivariate analyses for prognostic factors were performed using the Cox proportional hazard regression model. However, there was no significant difference in the univariate analysis of the TCGA cohort $(\mathrm{HR}=0.93$; 95\% CI: 0.64-1.35; $P=0.72$ ).

We then examined the LGR5 mRNA expression levels in the TCGA cohort when stage II/III patients were limited to those with histological grade 3 . There was no

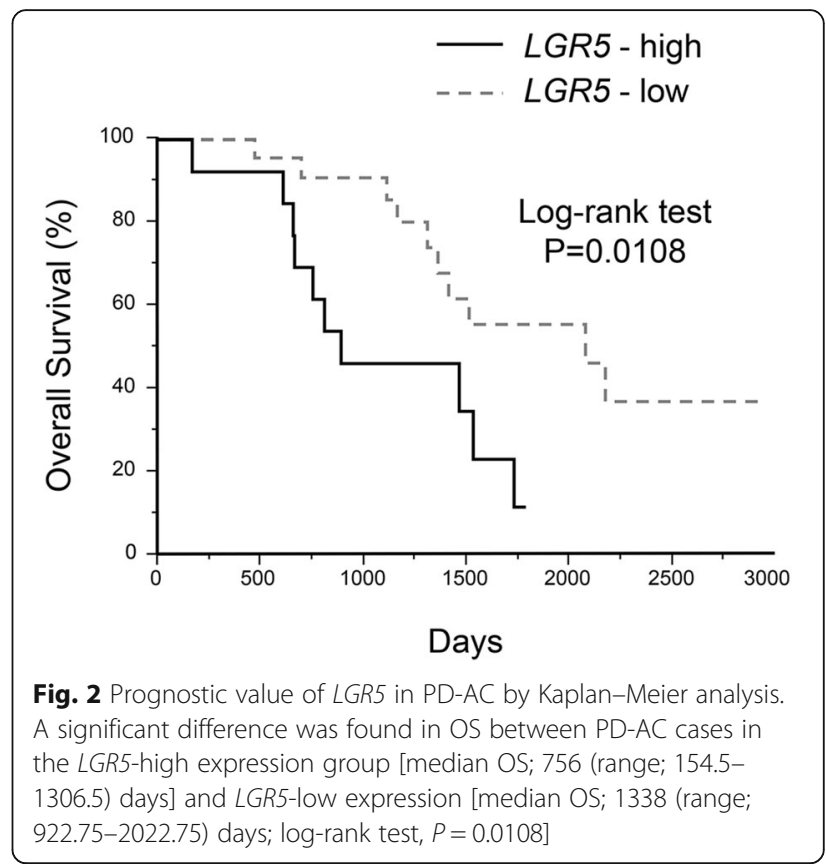

significant difference in OS between the LGR5 high and low groups (log-rank test, $P=0.7198)$. Univariate and multivariate analyses for prognostic factors were performed using the Cox proportional hazard regression model. However, there was no significant difference in the univariate analysis of the TCGA cohort $(\mathrm{HR}=1.08$; 95\% CI: $0.70-1.67 ; P=0.72$ ).

\section{Discussion}

LGR5 is an independent prognostic factor in PD-AC stages II and III. Although PD-AC has a poor prognosis [9], the related factors are not well understood. PD-AC exists as solid and non-solid subtypes. The prognosis of patients with the non-solid subtype with fibrosis is poor [12]. In our study, most PD-AC cases were non-solid, but no clear difference was observed in the prognosis of both non-solid and solid subtypes. LGR5 is also a promising gastric CSC marker, and high LGR5 expression in the poor prognosis group may suggest an involvement of CSCs in determining the prognosis. Therefore, LGR5 may be a therapeutic target in PD-AC and improve PDAC patient prognoses.

Migration ability and epithelial-mesenchymal transition (EMT) are increased in poorly differentiated gastric cancer [13]. Therefore, the poor prognosis of patients with high LGR5 expression may be related to the histological features of poorly differentiated cancer represented by enhanced migration ability, EMT-related protein expression, and LGR5 expression. Cancer cell migration is known to affect prognosis in gastric cancer [13]. Additionally, LGR5 expression, although not in the stomach, is related to migration ability and EMT [14]. In our study, 
Table 2 Univariate and multivariate analyses of prognostic factors for PD-AC

\begin{tabular}{|c|c|c|c|c|}
\hline \multirow[t]{2}{*}{ Factors } & \multicolumn{2}{|c|}{ Univariate analysis } & \multicolumn{2}{|c|}{ Multivariate analysis } \\
\hline & $\mathrm{HR}(95 \% \mathrm{Cl})$ & $P$-value & $\mathrm{HR}(95 \% \mathrm{Cl})$ & $P$-value \\
\hline Age: $>74$ years vs $\leq 74$ years & $1.49(0.57-3.69)$ & 0.3986 & & \\
\hline Sex: Male vs Female & $0.65(0.26-1.69)$ & 0.3596 & & \\
\hline Vascular invasion: Present vs Absent & $3.07(0.64-55.22)$ & 0.1936 & & \\
\hline TIL: Low vs High & $1.94(0.77-4.86)$ & 0.1575 & & \\
\hline MSI: Present vs Absent & $2.07(0.79-5.39)$ & 0.1338 & & \\
\hline TNM stage: II vs III & $0.39(0.15-0.97)$ & 0.0425 & $0.36(0.13-0.89)$ & 0.026 \\
\hline LGR5: High vs Low & $3.18(1.24-8.39)$ & 0.0161 & $3.48(1.36-9.22)$ & 0.01 \\
\hline
\end{tabular}

the correlation between vascular invasion and high $L G R 5$ expression may support an association of $L G R 5$ expression with EMT. High LGR5 expression and EMT were reported to be correlated in gastric cancer $[15,16]$. Furthermore, Zhang et al. reported that the LGR5 ligand RSPO2 promotes EMT in gastric cancer cells by activating WNT/ $\beta$-catenin signaling via LGR5 [17]. Therefore, elucidation of the relationship between LGR5 and RSPO2 in PD-AC may lead to the development of new therapeutic methods and an improved prognosis for PD-AC.

$L G R 5$ expression in the tumors of various organs has been widely investigated, mainly using immunostaining. Although some reports have indicated that high LGR5 expression is associated with a poor prognosis [18, 19], others have used RNAscope, which is considered to be more reliable $[20,21]$, and have reported that high $L G R 5$ expression correlates with a good prognosis specifically in pancreatic ductal adenocarcinoma [21]. We previously utilized RNAscope and showed that high LGR5 expression might be a poor prognostic factor in breast cancer [22].

Several reports have investigated LGR5 expression in gastric cancer. One RNAscope-based study did not uncover any difference in the OS of gastric cancer patients with varying expression of LGR5 [23]. In other study of gastric cancer, high immunostaining scores for $L G R 5$ were significantly associated with an increased risk of death [24]. Taken together, these data indicate that the role of LGR5 may be tissue-specific, and that its protein and mRNA regulation may be more complex than it seems.

While in our study, higher levels of LGR5 expression were associated with adverse prognosis, no significant differences in the overall survival were detected in TCGA cohort. This discrepancy might be due to ethnic or technical differences.

In addition, $\mathrm{Bu}$ et al. reported that $L G R 5$ expression is associated with a favorable prognosis, although this was limited to stages I and II [25]. Furthermore, LGR5 expression was associated with a high stage and lymph node metastasis [23]. $\mathrm{Xi}$ et al. reported that high $L G R 5$ expression is associated with poorly differentiated cancer [26]. However, $\mathrm{Bu}$ et al. reported that LGR5 is highly expressed in well-differentiated cancer [25]. Additionally, in studies using RNAscope, high LGR5 expression is correlated with well-differentiated cancer [27]. However, there is no comparison between LGR5 expression and prognosis in PD-AC using RNAscope, and the association between high LGR5 expression and poor prognosis in poorly differentiated cancer is a new finding.

The tendency for low LGR5 expression in EBVpositive tumors may be a feature of EBV-associated gastric cancer. According to a novel molecular pathological classification [28], EBV-associated gastric cancer is recognized as a distinct type of gastric cancer with a good prognosis [29]. The Cancer Genome Atlas Research Network reports that gastric cancer is divided into four types [28]. Therefore, to accumulate further knowledge in the future, it is necessary to analyze LGR5 expression in each type.

Our study has some limitations. This study included a relatively small sample size, which may have led to unreliable estimates. LGR5 expression and migration must be investigated in cultured cells; additionally, LGR5 expression must be analyzed in EBV-infected cells.

\section{Conclusions}

The association of LGR5 expression and patient prognosis in poorly differentiated gastric cancer may be applicable to the development of LGR5 targeted therapy and prognostic markers, but further study is desired.

\section{Abbreviations \\ CSC: Cancer stem cell; LGR5: Leucine-rich repeat-containing G-protein- coupled receptor 5; PD-AC: Poorly differentiated adenocarcinoma; TMA: Tissue microarray; TILs: Tumor-infiltrating lymphocytes; OS: Overall survival; EMT: Epithelial-mesenchymal transition}

\section{Acknowledgments}

We are grateful to Yukihiro Kobayashi, Masanobu Momose, Yasuyo Shimojo, Naoko Ogiwara, Akiko Inamura, Chitoshi Arai, Yasuhiro Kinugawa, Marina Nuno, Kanade Wakabayashi, Naoko Yamaoka, and Tomoya Hachisu at Shinshu University Hospital for their excellent technical assistance. We are grateful to Takenari Nakata (Department of Surgery, Nagano Matsushiro General Hospital), Yukiko Kusama (Department of Pathology, Nagano 
Municipal Hospital), Yukihiko Karasawa (Department of Surgery, Showa-Inan General Hospital), and Hisashi Shimojo (Department of Pathology, Aizawa Hospital) for collaboration on this work. We thank Nicole Okoh, PhD, and Melissa Crawford, PhD, from Edanz Group (https://en-author-services.edanz.com/ ac) for editing a draft of this manuscript.

\section{Authors' contributions}

TE participated in the design of the study, performed the pathological analysis, and drafted the manuscript. TU, SK, SA, and MI helped with the pathological analysis. TU performed the statistical analysis. TN and YK conducted immunohistochemistry. TE and US examined the clinical data of cases. $\mathrm{HO}$ and TU critically revised the draft for important intellectual content. All authors have read and approved the manuscript.

\section{Funding}

This study was partially supported by the Hokuto Foundation for Bioscience (grant awarded to T.U.). The funding body had no role in the study design, collection, analysis, or interpretation of data or manuscript writing. The authors declare no conflicts of interest.

\section{Availability of data and materials}

All data generated and analyzed during the current study are available from the corresponding author on reasonable request.

\section{Ethics approval and consent to participate}

This study was approved by the Ethics Committee of Shinshu University School of Medicine (Approval Code: 4088). The requirement of informed consent was waived, and an opt-out method was used because of the retrospective design of the study. The investigation was conducted in compliance with the Helsinki Declaration.

\section{Consent for publication}

Not applicable.

\section{Competing interests}

The authors declare that they have no competing interests.

\section{Author details}

${ }^{1}$ Department of Surgery, Shinshu University School of Medicine, Matsumoto, Japan. ${ }^{2}$ Department of Laboratory Medicine, Shinshu University School of Medicine, 3-1-1 Asahi, Matsumoto 390-8621, Japan. ${ }^{3}$ Department of Biomedical Laboratory Medicine, Shinshu University School of Medicine, Matsumoto, Japan.

Received: 6 May 2020 Accepted: 15 February 2021

Published online: 06 March 2021

\section{References}

1. Bray F, Ferlay J, Soerjomataram I, Siegel RL, Torre LA, Jemal A. Global cancer statistics 2018: GLOBOCAN estimates of incidence and mortality worldwide for 36 cancers in 185 countries. CA Cancer J Clin. 2018;68:394-424.

2. Bekaii-Saab T, El-Rayes B. Identifying and targeting cancer stem cells in the treatment of gastric cancer. Cancer. 2017;123:1303-12.

3. Brungs D, Aghmesheh M, Vine KL, Becker TM, Carolan MG, Ranson M. Gastric cancer stem cells: evidence, potential markers, and clinical implications. J Gastroenterol. 2016;51:313-26.

4. Wang T, Ong CW, Shi J, Srivastava S, Yan B, Cheng CL, et al. Sequentia expression of putative stem cell markers in gastric carcinogenesis. $\mathrm{Br} J$ Cancer. 2011;105:658-65.

5. Li XB, Yang G, Zhu L, Tang YL, Zhang C, Ju Z, et al. Gastric Lgr5(+) stem cells are the cellular origin of invasive intestinal-type gastric cancer in mice. Cell Res. 2016;26:838-49.

6. Leushacke M, Tan SH, Wong A, Swathi Y, Hajamohideen A, Tan LT, et al. Lgr5-expressing chief cells drive epithelial regeneration and cancer in the oxyntic stomach. Nat Cell Biol. 2017;19:774-86.

7. Van Cutsem E, Sagaert X, Topal B, Haustermans K, Prenen H. Gastric cancer. Lancet. 2016;388:2654-64.

8. Nakagawa N, Kanda M, Ito S, Mochizuki Y, Teramoto H, Ishigure K, et al. Pathological tumor infiltrative pattern and sites of initial recurrence in stage II/II gastric cancer: propensity score matching analysis of a multiinstitutional dataset. Cancer Med. 2018;7:6020-9.
9. Adachi Y, Yasuda K, Inomata M, Sato K, Shiraishi N, Kitano S. Pathology and prognosis of gastric carcinoma: well versus poorly differentiated type. Cancer. 2000;89:1418-24.

10. Nakajima T, Uehara T, Iwaya M, Kobayashi Y, Maruyama Y, Ota H. Characterization of LGR5 expression in poorly differentiated colorectal carcinoma with mismatch repair protein deficiency. BMC Cancer. 2020;20:319.

11. Ropponen KM, Eskelinen MJ, Lipponen PK, Alhava E, Kosma VM. Prognostic value of tumour-infiltrating lymphocytes (TILs) in colorectal cancer. J Pathol. 1997;182:318-24.

12. Kunisaki C, Akiyama H, Nomura M, Matsuda G, Otsuka Y, Ono HA, et al. Clinicopathological properties of poorly-differentiated adenocarcinoma of the stomach: comparison of solid- and non-solid-types. Anticancer Res. 2006;26:639-46.

13. Ueta K, Otowa Y, Kakeji Y, Hirashima M. PROX1 is associated with Cancer progression and prognosis in gastric Cancer. Anticancer Res. 2018;38:6139-45.

14. Zhang J, Cai H, Sun L, Zhan P, Chen M, Zhang F, et al. LGR5, a novel functional glioma stem cell marker, promotes EMT by activating the Wnt/ beta-catenin pathway and predicts poor survival of glioma patients. J Exp Clin Cancer Res. 2018;37:225.

15. Du M, Zhuang Y, Tan P, Yu Z, Zhang X, Wang A. microRNA-95 knockdown inhibits epithelial-mesenchymal transition and cancer stem cell phenotype in gastric cancer cells through MAPK pathway by upregulating DUSP5. J Cell Physiol. 2020;235:944-56.

16. Wang B, Chen Q, Cao Y, Ma X, Yin C, Jia Y, Zang A, Fan W, Papaccio G. LGR5 is a gastric cancer stem cell marker associated with stemness and the EMT signature genes NANOG, NANOGP8, PRRX1, TWIST1, and BMI1. PLOS ONE. 2016;11(12):e0168904.

17. Zhang H, Han X, Wei B, Fang J, Hou X, Lan T, et al. RSPO2 enhances cell invasion and migration via the WNT/beta-catenin pathway in human gastric cancer. J Cell Biochem. 2019;120:5813-24.

18. Takahashi H, Ishii H, Nishida N, Takemasa I, Mizushima T. Ikeda met al. Significance of Lgr5(+ve) cancer stem cells in the colon and rectum. Ann Surg Oncol. 2011;18:1166-74.

19. Chen W, Fu Q, Fang F, Fang J, Zhang Q, Hong Y. Overexpression of leucinerich repeat-containing $\mathrm{G}$ protein-coupled receptor 5 predicts poor prognosis in hepatocellular carcinoma. Saudi J Biol Sci. 2018;25:904-8.

20. Martin ML, Zeng Z, Adileh M, Jacobo A, Li C, Vakiani E, et al. Logarithmic expansion of LGR5(+) cells in human colorectal cancer. Cell Signal. 2018;42: 97-105.

21. Kuraishi Y, Uehara T, Kobayashi Y, Nakajima T, Watanabe T, Shimizu A, et al. Correlation of clinicopathological features and leucine-rich repeatcontaining G-protein-coupled receptor 5 expression in pancreatic ductal adenocarcinoma. Pathol Res Pract. 2019;215:152623.

22. Ogasawara S, Uehara T, Nakajima T, Iwaya M, Maeno K, Tsuchiya S, et al. Correlation of clinicopathological features and LGR5 expression in triplenegative breast cancer. Ann Diagn Pathol. 2020;46:151491.

23. Jang BG, Lee BL, Kim WH. Prognostic significance of leucine-rich-repeatcontaining G-protein-coupled receptor 5, an intestinal stem cell marker, in gastric carcinomas. Gastric Cancer. 2016;19:767-77.

24. Huang T, Qiu X, Xiao J, Wang Q, Wang Y, Zhang Y, et al. The prognostic role of Leucine-rich repeat-containing G-protein-coupled receptor 5 in gastric cancer: A systematic review with meta-analysis. Clin Res Hepatol Gastroenterol. 2016:40:246-53.

25. Bu Z, Zheng Z, Zhang L, Li Z, Sun Y, Dong B, et al. LGR5 is a promising biomarker for patients with stage I and II gastric cancer. Chin J Cancer Res. 2013;25:79-89.

26. Xi HQ, Cai AZ, Wu XS, Cui JX, Shen WS, Bian SB, et al. Leucine-rich repeatcontaining G-protein-coupled receptor 5 is associated with invasion, metastasis, and could be a potential therapeutic target in human gastric cancer. Br J Cancer. 2014;110:2011-20.

27. Jang BG, Lee BL, Kim WH. Distribution of LGR5+ cells and associated implications during the early stage of gastric tumorigenesis. PLoS One. 2013:8:e82390.

28. Cancer genome atlas research $\mathrm{N}$ comprehensive molecular characterization of gastric adenocarcinoma. Nature. 2014;513:202-9.

29. Liu X, Liu J, Qiu H, Kong P, Chen S, Li W, et al. Prognostic significance of Epstein-Barr virus infection in gastric cancer: a meta-analysis. BMC Cancer. 2015;15:782.

\section{Publisher's Note}

Springer Nature remains neutral with regard to jurisdictional claims in published maps and institutional affiliations. 